\title{
A Research Review on Job Shop Scheduling Problem
}

\author{
Yingchen $\mathrm{Yu}^{1}$ \\ ${ }^{1}$ School of Economics and Management Beijing Jiaotong University Beijing, China
}

\begin{abstract}
In recent years, the manufacturing industry has developed rapidly with fierce competition. Manufacturing enterprises are faced with the challenge of on-time delivery, multiple product choices and quick response to order modification. Reasonable and efficient production scheduling determines whether the enterprise can continue to survive. Therefore, the study of job shop scheduling problem has practical significance. This paper summarizes the research development and current situation of job shop scheduling problem, classifies the existing research methods, and discusses the future research direction of job shop scheduling problem.
\end{abstract}

\section{INTRODUCTION}

Job shop scheduling is the core of job planning for workshop production process, which makes manufacturing industry realize intelligence, automation and informatization. Job shop scheduling problem (JSP) is the core part of production management in job shop system. The efficient completion of production tasks requires reasonable job shop scheduling. Therefore, the study of job shop scheduling problem has very important theoretical significance and application value. Job shop scheduling problem is one of the typical complex scheduling problems with high complexity and difficulty. It has a strong engineering application background. It means that according to the single or multiple production objectives and the workshop environment status, under the premise of satisfying the constraint conditions, the processing sequence of the jobs is reasonably sorted, and the sequence obtained determines the start and end of each job process End time to optimize one or more scheduling performance metrics. This paper reviews the research of job shop scheduling problem, and then looks forward to the research direction of job shop scheduling problem.

\section{JSP overview}

Job shop scheduling problem can be described as $n$ jobs are processed on $\mathrm{m}$ machines. The set of all jobs is $\mathrm{j}=\{\mathrm{Ji}$ $\mid(\mathrm{I}=1,2, \ldots)$ The set of all machines is $\mathrm{m}=\{\mathrm{mk} \mid(\mathrm{k}=1,2, \ldots)$ The constraints to be satisfied in the process of workpiece processing are as follows:

(1) At the same time, the workpiece can only be processed on one machine at most;

(2) The processing process should meet the requirements of the process route of the corresponding workpiece;

(3) At the same time, a machine can only process one workpiece at most;

(4) Once the process is started on the machine, it can not be interrupted until it is finished.

At first, the job shop scheduling problem was studied as a mathematical problem, focusing on theory, mostly using the optimization methods of mathematics and operational research, in order to obtain the global optimal solution. In 1954, Johnson ${ }^{[1]}$ was the first to study the flow shop scheduling of two machines processing in sequence. Since 1980s, with the continuous development of computer technology and intelligent algorithm, scholars have applied it to the solution of job shop scheduling problem, and a series of research methods have appeared. At present, the main method to solve job shop scheduling problem is to use intelligent algorithm to solve it. Table 1 shows the main research methods of job shop scheduling problem.

TABLEI. MAIN RESEARCH METHODS OF JOB SHOP SCHEDULING PROBLEM

\begin{tabular}{|c|c|}
\hline Precise method & Mathematical programming Branch and bound method \\
\hline \multirow{3}{*}{ Approximate method } & Mobile bottleneck method \\
\cline { 2 - 2 } & System simulation method \\
\cline { 2 - 2 } & Intelligent algorithm \\
\hline
\end{tabular}

\section{JSP Research methodology system}

\subsection{Mathematical programming}

Early researchers used accurate methods to solve scheduling problems, mainly based on Mathematics and operational research, including mathematical programming, branch and bound method, decomposition method and so on. However, these precise methods can only get the optimal solution in theory. Due to the complexity of calculation, large amount of calculation, 
time-consuming and other reasons, these precise solutions are difficult to be applied to practical problems.

\subsection{Lagrangian Relaxation}

As an approximate algorithm for solving complex optimization problems, Laplace relaxation technique has attracted extensive attention in recent years due to its ability to obtain high-quality suboptimal solutions in a short time and to evaluate its performance. The IP of the integer programming problem is transformed into Lagrangian relaxation according to Lagrange relaxation method to expand the solution space of the problem. The Laplace relaxation algorithm obtains that the feasible solution of the problem is the upper bound of the optimal solution of the original problem. Then the solution of the corresponding dual problem of the integer programming problem is obtained, which is the lower bound of the optimal solution of the original problem. Finally, the solution is transformed into the feasible solution of the original problem. It can be used to solve the single machine scheduling and multiple parallel machine scheduling problems.

\subsection{System simulation method}

System simulation method is to collect data through the operation of simulation model, and use these data to analyze the performance and state of the actual system, and adopt appropriate control and scheduling method for the system according to their own experience. Although the system simulation method can provide users with a test opportunity, it has the following problems:

(1) It is difficult to get general regularity;

(2) The cost of simulation is very high;

(3) The accuracy of simulation is greatly limited by the experience and judgment of users.

\subsection{Mobile bottleneck method}

The moving bottleneck method was proposed by Adams et al. ${ }^{[2]}$ in 1988, and the optimal solution of $\mathrm{ft} 10$ example was obtained successfully. They proposed a method to determine the bottleneck degree of machines and give priority to the machines with the highest bottleneck degree. In order to sort the machines, the single machine scheduling problem is solved after relaxing the original problem for each machine that has not been sorted, that is, only one machine is considered at a time. The time span obtained from the single machine scheduling is used to determine the priority of the machines that have not been sorted. The machine with the highest priority has the highest bottleneck degree. Every time a new machine is finished, those machines that have been ordered are re optimized. It is possible to solve the single machine scheduling problem again to make the final solution better. When the order of all machines is determined. It is easy to find the scheduling time span. However, the algorithm of mobile bottleneck method is complex and the calculation time is too long, so it is difficult to be applied to the actual job shop scheduling problem.

\subsection{Artificial intelligence method}

Constraint satisfaction technology. Constraint satisfaction technology is an intelligent approximation method for solving large-scale combinatorial optimization problems by reducing the effective scale of search space by using constraints. Pesch et al. Decompose the whole problem into a single machine scheduling problem based on constraint propagation technology, and deeply study the special structure of the famous ft10 example.

Neural network. There are three ways to apply neural network to job shop scheduling. One is to use its parallel computing ability to solve the optimal scheduling problem to overcome the NP difficulty of scheduling. The second is to make use of its learning ability, so that the network can correctly select the appropriate scheduling strategy and evaluation index according to the production characteristics. The third is to describe scheduling constraints or scheduling strategies to achieve feasible or suboptimal scheduling of production processes.

Expert system and multi-agent technology. Expert system is a kind of computer program system which is composed of knowledge base and inference engine to simulate human experts to solve domain problems. Because of the limited knowledge and weak ability of expert system, people began to develop distributed artificial intelligence system. Like expert system, multiagent technology has the bottleneck problem of building knowledge base.

\subsection{Intelligent algorithm solution}

After hundreds of millions of years of natural selection, survival of the fittest, inheritance and creation, nature has formed beautiful, complex and diverse life phenomena and natural wonders, which contains rich information processing mechanisms, providing an inexhaustible source of wisdom for the intelligent process of human society. It is through the study of the rich information processing mechanism contained in it that a variety of computational intelligence algorithms have been produced. At present, intelligent algorithms have been successfully applied to solve the job shop scheduling problem, such as genetic algorithm, tabu search algorithm, simulated annealing algorithm, etc.

\subsection{1 genetic algorithm}

Genetic algorithm is a random search algorithm based on natural selection and natural genetic mechanism in biological world. It has many advantages, especially its strong global search ability, self-organization, selflearning and so on. Davis ${ }^{[3]}$ first applied genetic algorithm to job shop scheduling problem based on priority table. After that, many researchers have carried out a lot of research by improving the performance of genetic algorithm. Goncalves [4] proposed a hybrid genetic algorithm with random key coding and local heuristic search to solve job shop scheduling problem. Zhang ${ }^{[5]}$ proposed to use fuzzy logic to adaptively adjust the mutation rate and crossover rate in GA instead of fixed 
values. The k-means algorithm is used to adjust the mutation rate and crossover rate, and improve the performance of the algorithm. Yildirim ${ }^{[6]}$ and others proposed a mathematical model to minimize energy consumption and reduce the total completion time of a single machine, and used multi-objective genetic algorithm to obtain a group of approximate non dominated alternatives. Goncalves et al. ${ }^{[7]}$ proposed a random key genetic algorithm for solving JSP problems, which improved the current optimal solutions of 57 benchmark JSP problems. The principle and operation of genetic algorithm is simple, and it has strong generality. It is not restricted by restrictive conditions. It has implicit parallelism and global solution space searching ability, But premature and low search efficiency are the main shortcomings of genetic algorithm. Through the above research, we can see that researchers are constantly improving the genetic algorithm to solve the job shop scheduling problem and achieved a series of results.

\subsection{2 tabu search algorithm}

Tabusarch is a heuristic algorithm for finding approximate optimal solutions of combinatorial problems. It was first proposed by Glover and Hansen in 1986, and finally developed and perfected by Glover. It can find the optimal solution of some instances in the scheduling problem. Tabu search can find the optimal solution of many scheduling problems. However, tabu search has to face many problems, such as initial solution problem, neighborhood structure problem, search strategy and the length of tabu table. Taillard ${ }^{[8]}$ first applied tabu search algorithm to solve job shop scheduling problem. Nowicki et al. ${ }^{[9]}$ proposed a tabu search algorithm based on critical path and block structure to solve JSP problem. On this basis, a more efficient i-tsab algorithm was proposed in $2002{ }^{[10]}$. In 2007, Zhang [11] proposed a tabu search algorithm based on N7 neighborhood structure. In 2008, tabu search and simulated annealing algorithm were further mixed, and new solutions were obtained in some benchmark examples of JSP problem ${ }^{[12]}$. In 2012, nasiri et al. ${ }^{[13]}$ mixed the Global Balanced search algorithm and tabu search algorithm to solve JSP problems, and improved the current optimal solution of 19 benchmark JSP problems. In 2014, Peng et al. ${ }^{[14]}$ solved JSP problems based on the tabu search algorithm of N7 neighborhood structure, improved the current optimal solution of 49 benchmark JSP problems, and promoted the solution algorithm of JSP problem to a new height. In 2019, Ibrahim $^{[15]}$ et al. applied tabu search algorithm, geometric particle swarm optimization algorithm, genetic algorithm, simulated annealing algorithm and I-L algorithm for the job shop scheduling problem with two identical parallel machines as the optimization objective. Finally, for the larger and more complex scheduling problems, tabu search algorithm can obtain a better average completion time, and is closer to the lower limit of time. The efficiency of tabu search algorithm is better than that of tabu search algorithm. But tabu search algorithm has to face many problems, such as initial solution problem, neighborhood structure problem, search strategy and the length of tabu table. Only by solving these problems well can tabu search algorithm be applied better.

\subsection{3 simulated annealing algorithm}

The original idea of simulated annealing algorithm is proposed by metropolis, and its optimization idea is to refer to the process of physical annealing. Matsuo ${ }^{[16]}$ and van laarhoven ${ }^{[17]}$ have applied simulated annealing method to job shop scheduling problem. Pemera ${ }^{[18]}$, jolai ${ }^{[19]}$ and others used the simulated annealing algorithm to solve the flow shop scheduling problem with the optimization objectives of minimizing the completion time and the number of jobs with delayed completion, and compared with the previous results, the effectiveness of the algorithm was proved. In 2016, Kashif ${ }^{[20]}$ and others used the fast simulated annealing algorithm and quenching hybrid algorithm to find the global optimal solution, and avoid falling into the local optimization. With the optimization goal of minimizing the completion time, the job shop scheduling problem was optimized and compared with 88 benchmark examples, and the optimal solution was 45 within a reasonable time Finally, it is proved that the algorithm can find the optimal solution of the job shop scheduling problem effectively. Because simulated annealing algorithm requires high initial temperature and slow cooling speed, it can converge to the global optimal value. It is a kind of serial optimization algorithm with low computational efficiency, so it should be improved generally.

\subsection{4 other intelligent algorithms}

In addition to the above algorithms, there are also some intelligent algorithms to solve the job shop scheduling problem. Differential evolution algorithm is an evolutionary computing technology based on population difference, which was proposed by storm and price. Ponsich et al. ${ }^{[21]}$ mixed differential evolution algorithm with tabu search algorithm to solve job shop scheduling problem. In the 1990s, Dorigo et al. Proposed ant colony algorithm by observing and studying the foraging behavior of ants. Cao ${ }^{[22]}$ and others used the global search performance of ant colony algorithm and the local search performance of tabu search algorithm to solve job shop scheduling problem. Artificial bee colony algorithm is a new swarm intelligence optimization algorithm proposed in 2005 . In 2014 , Zhang et al. ${ }^{[23]}$ proposed a new artificial bee colony algorithm for JSP problem with weighted tardiness sum as optimization objective.

\section{4 summary and Prospect}

Reasonable job shop scheduling is the key to improve the efficiency of production system, improve the utilization of system resources, and enhance the core competitiveness of enterprises. Meanwhile, the job shop scheduling problem has its complexity and representativeness, which has been concerned by researchers at home and abroad in recent decades. From the above research and development of job shop scheduling problem, we can see that the solution of 
job shop scheduling problem mainly includes mathematical programming method, system simulation method and intelligent algorithm. Although each method has made great progress, there are still some unsolved problems. Although the accurate algorithm has advantages in solving accuracy, its solving time is too long; the approximate algorithm has short solving time, but the quality of the solution is difficult to guarantee. Therefore, the intelligent algorithm with short solving time and high precision is the research hotspot of scholars. At present, the main research direction of researchers is to improve and upgrade the existing research methods to obtain better scheduling results, but at present, there are few solutions to the inherent problems of job shop scheduling, so there is no systematic solution to the problem. The author thinks that the future research focuses on the following aspects:

(1) Fusion of different algorithms. The mechanism of different algorithms is different, so each algorithm has its advantages and disadvantages. In order to solve the job shop scheduling problem more efficiently, the existing two or more algorithms are integrated to give full play to their own advantages.

(2) The integration of different research methods. By analyzing and summarizing the advantages of each research method system and combining their complementary points, we can make them complement each other in solving job shop scheduling problems, and achieve efficient and accurate results.

(3) The theoretical research is connected with the actual job shop scheduling. The research of job shop scheduling problem is ultimately to serve the solution of practical problems. When establishing the model of job shop scheduling problem, the constraint conditions should be set according to various conditions in actual production, and the efficient algorithm in line with the actual production should be studied.

(4) The innovation of the original method. Creating a new simulation system, mining new algorithms according to new principles.

\section{Reference}

1. JOHNSON S M. Optimal two and three-stage production schedules with setup times included[J]. Naval Research Logistics Quarterly,1954,1(1):61 - 68.

2. ADAMS J,BALAS E,ZAWACK D. The shifting bottleneck procedure for job shop scheduling[J]. Management Science,1988,34(3):391 - 401.

3. DAVIS L. Job shop scheduling with genetic algorithms[C]/ /Proceedings of an International Conference on Genetic Algorithms and Their Applications, 1985:136 - 140.

4. GONCALVES J F,MAGALHAES MENDES J J,RESENDE M G C.A hybrid genetic algorithm for the job shop scheduling problem [J]. European Journal of Operational Research,2005,167 (1):77-95

5. ZHANG C Y,RAO Y Q,LI P G. An effective hybrid genetic algorithm for the job shop scheduling problem[J].International Journal of Advanced Manufacturing Technology,2008,39:965 - 974.
6. Yildirim M B., Mouzon G. Single-Machine Sustainable Production Planning to Minimize Total Energy Consumption and Total Completion Time Using a Multiple Objective Genetic Algorithm[J]. IEEE Transactions on Engineering Management. 2012,59(4):585-597

7. GONCALVES J F,RESENDE M G C. An extended akers graphical method with a biased randon-key genetic algorithm for job shop scheduling $[\mathrm{J}]$.International Transactions in Operational Research,2014,21(2):215 - 246.

8. Taillard E D. Parallel taboo search techniques for the job shop scheduling problem[J]. ORSA Journal on Computing. 1994,2(6):108-117.

9. NOWICKI E,SMUTNICKI C.A fast taboo search algorithm for the job shop problem[J]. Management Science, 1996,42 (6):797-813

10. NOWICKI E,SMUTNICKI C. Some new tools to solve the job shop problem[R]. Institute of Engineering Cybernetics, Wroclaw University of Technology,2002.

11. ZHANG C Y,LI P G,GUAN Z L,et al.A tabu search algorithm with a new neighborhood structure for the job shop scheduling problem[J].Computers \& Operations Research,2007,34(11):3229-3242.

12. ZHANG C Y,LI P G,RAOYQ,et al.A very fast TS /SA algorithm for the job shop scheduling problem [J].Computers\&Operations Research,2008,35(1):282 - 294.

13. NASIRI M M,KIANFAR F.A GES/TS algorithm for the job shop scheduling[J]. Computers \& Industrial Engineering,2012,62(4):946 - 952.

14. PENG B,LU Z,CHENG T C E. A tabu search/path relinking algorithm to solve the job shop scheduling problem[J].Computers \& Operations Research,2015,53:154 - 164.

15. Alharkan I., Saleh M., Ghaleb M A., et al. Tabu search and particle swarm optimization algorithms for two identical parallel machines scheduling problem with a single server[J]. Journal of King Saud University Engineering Sciences. 2019.

16. MATSUO H,SUH C J,SULLIVAN R S. A controlled search simulated annealing method for the general job-shop scheduling problem[R]. Graduate School of Business, University of Texas at Austin, 1988.

17. VAN LAARHOVEN $P$ J M,AARTS E H L,LENSTRA J K. Job shop scheduling by simulated annealing [J]. Operations Research,1992,40( 1) : 113 -125 .

18. Jarosław P., Czesław S., Dominik Ż. Optimizing Bicriteria Flow Shop Scheduling Problem by Simulated Annealing Algorithm[J]. Procedia Computer Science. 2013,18:936-945.

19. Jolai F., Asefi H., Rabiee M., et al. Bi-objective simulated annealing approaches for no-wait two-stage flexible flow shop scheduling problem[J]. Scientia Iranica. 2013,20(3):861-872.

20. Akram K., Kamal K., Zeb A. Fast simulated annealing 
hybridized with quenching for solving job shop scheduling problem[J]. Applied Soft Computing. 2016,49:510-523.

21. PONSICH A,COELLO COELLO C A. A hybrid differential evolution-tabu search algorithm for the solution of job-shop scheduling problems[J]. Applied Soft Computing,2013,13( 1) : 462 - 474.

22. CAO Y,SONG X,ZHANG Y,et al. Application of
ACOTS hybrid algorithm for job shop scheduling problems[C]/ /2012 Proceedings of International Conference on Modelling,Identification \&Control,IEEE,2012: 289 - 293.

23. ZHANG R,SONG S,WU C. A hybrid artificial bee colony algorithm for the job shop scheduling problem[J]. International Journal of Production Economics,2013,141( 1) : 167 - 178. 